(C) 1971 IEEE. Personal use of this material is permitted. However, permission to reprint/republish this material for advertising or promotional purposes or for creating new collective works for resale or redistribution to servers or lists, or to reuse any copyrighted component of this work in other works must be obtained from the IEEE.

\title{
HIGH CHARGE STAII' HEAVY ION SOURCES
}

\author{
J.R.J. Bennett ${ }^{*}$ \\ High Energy Physics Laboratory \\ Stanford University, Stanford, California, U.S.A.
}

\section{Surmary}

A review is given of existing sources of highly charged heavy ions. The performance of the sources, predominantly of the P.I.G. type, are summarized and compared. Some mechanisms for the production of high charge states are described. There is a brief description of new developments, particularly of devices using ion trapping.

\section{Introduction}

Tremendous interest is presently being centred on nuclear reactions with energetic heavy particles (up to uranium). Unfortunately, for ions heavier than about argon existing accelerators ${ }^{1}$ are unable to attain the energies of $8-10 \mathrm{MeV} /$ nucleon necessary for these reactions because the ion sources do not produce ions sufficiently highly charged. A great many new prom posals (see, for example, Ref. 1) have been made to build larger accelerators. The majority of the prom posed accelerators consist of separated sector cycio trons with large tandem Van de Graaffs as injectors. The Berkeley Hilac is being rebuilt, ${ }^{2}$ the $310 \mathrm{~cm}$ Dubna cyclotron is to be enlarged, 3 and a large lipear accelerator is to be constructed in Germany. 4 These new machines use ion sources essentially unchanged from those developed initially nearly two decades ago.

It is clear that there is a pressing need for an ion source which will produce the required charge states for use with existing accelerators. A charge (in electron units) to mass (in a.m.u.) ratio of about 0.35 would allow most of the modern isochronous cyclotrons to achieve $10 \mathrm{MeV} /$ nucleon. The large Indiana separated sector cyclotron ${ }^{5}$ at present under construction will not be able to reach this energy for the heavier ions but could do so if ions of charge-to-mass ratio of 0.2 were available. New sources might well be expensive to develop but could in the end be a more economical solution than building large accelerators. An alternative is to develop new accelerators not requiring highly charged ions such as the electron ring accelerator. 6 Another solution could be the superconducting linear accelerator which is expected? to be relatively inexpensive even with existing ion sources.

It is the purpose of this paper to review and summarize the performance of existing multiply charged heavy ion sources and to indicate some of the new developments.

\section{Ionization Mechanisms}

There are many ways in which atoms can be ionized, but the two of most importance to be considered here are electron collisions and stripping.

\section{Stripping}

If an energetic ion is passed through a small quantity of matter, electrons will be stripped off, the number depending on the velocity, the atomic number, and the stripping medium. Figure 1 (taken from Ref. 1) shows the values, calculated from an empirical formula, of the average equilibrium charge state of an ion beam

*on leave of absence from the Rutherford HIgh Energy Laboratory, Chilton, Berks., England. after passing through a solid stripper as a function of the energy.

A number of accelerators, mainly heavy fon linacs and tandem Van de Graaffs, use stripping during the acceleration to increase the charge state. Hortig has proposed a charge exchange accelerator 9 using the stripping technique in a novel way. An Ion source consisting of a large separated sector cyclotron has been suggested by Jenkins. 10 Ions are accelerated stochastically in a poor vacuum to obtain "continuous" stripping.

\section{Ionization by Elcctron Collision}

Multiple ionization may occur at a single collision or as a result of repeated collisions producing successively higher states of ionization as one electron is removed at a time. The single collision processll occurs when a high energy electron removes or excites an inner shell electron causing Auger transitions and shake-off of several electrons. Cross sections have been measured by Schram et al; 12,13 the values decrease rapidly with the charge state. For example, the cross section for the formation of $\mathrm{A}^{+}$(see Fig. 2) is between 5 and 6 orders of magnitude lower than that for $\mathrm{A}^{+}$. However, the cross sections $14,15,16$ for removal of only one electron at a time from an ion decrease relatively slowly as the charge state increases. High electron energies of several thousand volts give optimum cross sections for the single collision process, but lower energies 15,16 may be used in stepwise ionizations.

\section{Ionization Devices}

The requirements of any device which is to produce reasonable quantities of highly charged ions are a high rate of ionization and a slow loss of ions due to recombination relative to the rate at which ions leave the ionizing region. If multiple ionization in single collisions is the only process, it is bound to result in small fractional currents of highly charged ions since the ratio of the fractions in each charge state varies as the ratio of the cross sections. However, a device known as EImo 17 is being investigated 18 in which multiply charged ions are expected to be produced by the single collision process. Postma ${ }^{19}$ has estimated that $20 \mathrm{\mu A} / \mathrm{cm}^{2}$ of $\mathrm{Xe}^{12+}$ should be obtained from Elmo.

With stepwise ionization it is essential for the ions to remain in the ionizing region for a long enough time to be repeatedly ionized. The product of the containment time and the ionizing electron flux is an important factor in determining the charge state that can be attained. Figure 3 shows the containment time calculated to achieve a mean charge state $z_{\text {eff }}$ for an electron flux of $6 \times 1020 / \mathrm{cm}^{2}$ for a device known as the HIPAC source. 20 Note the long time $(\sim 6 \mathrm{~s}$.) to reach $60+$ even with an electron current flux of nearly $100 \mathrm{~A} / \mathrm{cm}^{2}$. Other devices using the principle of stepwise lonization and containment have been suggested by Stix, 21 Trivelpiece, 22 and Williams. 23 In Dubna work is proceeding on the electron beam source, EBIS. ${ }^{24}$ Both EBIS and the HIPAC are discussed in a later section.

One of the newest fields of possible ion source development is with very high current pulsed electron beams. 25 In this case both acceleration and production of multiply charged heavy ions is achieved. The mechanism for production of the ions and their acceleration 
is not fully understood and it is too early to forecast how these devices may develop.

Up to now arc discharges working on the Penning Ionization Gauge 26 (F.I.G.) principle have been the basis of nearly all the multiply charged heavy ion sources. It has also been shown that the duoplasmatron? type of arc can provide multiply charged ions. The intense current spark discharge is well known from spectroscopic observations 28,29 to contain extremely high charge state lons. However, relatively few of these sources have been made.

Various plasmas used in fusion research have been found to contain highly charged ions; they have not been developed as ion sources. The irradiation of matter by very high intensity pulsed laser beams produces dense plasmas of highly ionized material and work is proceeding in their development into a practical ion source.

\section{P.I.G. Ion Sources}

\section{Source Characteristics}

Basically the P.I.G. source consists of two cathodes placed at the ends of a cylindrical hollow anode. Electrons oscillating between the cathodes are constrained from moving radially to the anode by a magnetic field of several thousand gauss aligned along the axis of the anode. In this way the electrons have a long path to the anode thereby increasing the chance of ionization. A plasma is formed filling the anode chamber. of course this is a very much simplified view of the complex and by no means fully understood processes 30 that occur in the P.I.G. discharge.

The production of multiply charged ions is considered to be predominantly by the stepwise mechanism. This is inferred 31 by the build up of the higher charge states with time and by the distribution of the currents among the various charge states which does not follow the values of the cross section for one step multiple ionization.

Ions may be obtained from the discharge through an aperture in either the anode (side extraction) or one of the cathodes (end extraction). The ion current that can be extracted varies with the aperture area 32 and the arc current. 33 Ion current densities of up to $0.5 \mathrm{~A} / \mathrm{cm}^{2}$ per ampere of arc current can be expected with end extraction and $0.05-0.1 \mathrm{~A} / \mathrm{cm}^{2}$ per ampere of arc current with side extraction.

There are several variations of the P.I.G. source. Some have hot cathodes emitting electrons thermionically whilst others have cold cathodes where electrons are produced only by secondary emission. The hot cathodes may be directly heated (filament), indirectly heated, or self-heated by the discharge. Another type of source frequently used in cyclotrons because of convenience has one cathode electrically isolated (flloating cathode or anticathode). In this case the other cathode must be hot; otherwise, the discharge will not strike.

The floating cathode design is not always reliable for the production of multiply charged ions because of the uncertainty of the potential attained by the isolated cathode. 34 The highly charged components of the ion beam begin to fall off rapidly if the potential of the floating cathode departs by more than a few volts from the potential of the other cathode. By careful adjustment of the arc conditions it is possible to make the potentials of the two cathodes the same, but the condition is not always stable.
There are three basic parameters of the arc: the arc voltage, the arc current, and the gas flow. The magnetic field is relatively unimportant. The arc parameters can be independently varied with directly or indirectly heated cathode sources at power levels where self-heating is not predominant. With cold and self-heated cathode sources the only independent parameters are the are voltage (or current) and the gas pressure. However, the arc characteristics 33,35 are quite different (see Figs. 4 and 5), the impedance being positive for the cold cathode and negative for the hot cathode source.

Pigarov and Morozov ${ }^{31}$ have studied the variation in the yield of the charge states for a number of gases in an indirectly heated cathode source. Independent control of the arc parameters was achieved even at high arc powers by pulsing the discharge. The general conclusion of the work is that a high arc current and voltage and a low gas pressure favours the production of high charge state ions. This result is found for all types of hot cathode sources. With cold cathode sources the yield36 of highly charged ions increases with power unti] an optimum is reached at an arc current of several amps.

One of the most critical parameters of hot and cold cathode sources is the gas pressure (Fig. 6). To obtain the maximum yield $33,35,37$ of highly charged ions the gas flow must be adjusted to a minimum value at which the discharge almost extinguishes.

With hot cathode soures it wonld ampear advantageous to increase the arc power further. This would also allow the arc to run at lower gas pressures. However, cathode erosion may be a limiting factor. With heavy ions a fonsiderable quantity of cathode material is sputtered ${ }^{38}$ into the discharge and can be a major contributor to the gas pressure. 37 The arc voltage and yield of high charge states then decreases. This problem is obviously not going to improve as the arc power is raised.

'The lifetime of the cathodes is governed by erom sion, sometimes to ten hours or less depending on the discharge conditions. Furthermore, the sputtered material deposits on the anode where it can sometimes build up and either short circuit the arc or constrict the discharge so that the plasma moves away from an extraction aperture in the anode thereby reducing the ion output.

\section{Performance}

Table I lists the performance of a number of P.I.G. sources operated with nitrogen, neon, argon, krypton, and xenon gases. Other types of sources are shown in the same table for comparison. Many of the sources have pulsed discharges and in these cases the pulse values of the parameters are listed. Note that the percentages of electric ion currents, not particle currents, are given. Where data is inadequate to calculate the percentages of current in the various charge states, the absolute value of the measured currents are given in parentheses. The table has been compiled from the literature and adapted to fit the form of presentation used here. The data is of bench tests of the sources. The best results producing the maximum fraction of ion current in the high charge states have been selected.

The first reference to P.I.G. sources used for multiply charged heavy ions is that of Jones and Zucker39 who developed two sources for nitrogen ions. The resolution of the spectra of the charge states is poor and some doubt must arise as to the accuracy of 
the results. Two years later Anderson and Ehlers 35 reported on the development of a cold cathode pulsed P.I.G. source (Fig. 7) for the Berkeley and Yale Hilacs. Development has been continued by Gavin. Following soon after, Morozov, Makov and Ioffe ${ }^{40}$ published an account of a source using an indirectly heated cathode. The source, shown in Fig. 8, has both cathodes connected electrically and the top cathode is heated by electrons accelerated to it from a hot filament. Thus the discharge can strike at a low arc voltage. At high arc powers where the cathodes are self-heated by the discharge, the indirect heating may be turned off. An unusual feature of the source is the square sectioned anode chamber. The Morozor design has been developed into a powerful source with the heavier elements and used very successfully with the $310 \mathrm{~cm}$ Dubna cyclotron.

From the table it can be seen that there is quite a spread in the performance of the different P.I.G. cources. However, it is possible to make some general observations on the production of the high charge state ions.

a) Side extraction is vastly superior to end extraction. Although Mineev and Kovpik, ${ }^{+1}$ using plasma expansion in extraction of the ions, report quite gond fractions of multiply charged ions, the spectrum shown in their publication indicates a very large current of molecular nitrogen ions. These ions are usually almost totaliy absent with side extraction sources and even with Bennett's end extraction source ${ }^{+2}$ less than $1 \%$ was found.

b) The pulsed cold cathode sources are superior to the hot cathode sources for the heavier elements (above argon). The reason for this is not clear but may be due to the higher arc voltages employed with cold cathode sources. However, hot cathode sources can also operate at high voltages, but Pasyuk et a. 37 find the higher voltages lcss favorable. Since the discharge current in the cold cathode source is much lower than that of the hot cathode sources the total ion current per unit area of extraction aperture is down in value by a large factor (as much as 10 in some cases). Making a large aperture in the source can compensate for this if the gas flow is not excessive. In the tables the actual currents of $\mathrm{Kr}^{10+}$ and $\mathrm{Xe} \mathrm{I}^{\mathrm{l}}$ obtained from the sources of Pasyuk 37 and Gavin 43 are shown in brackets. (The extraction area of Pasyuk's source was a little smaller than that of Gavin's.) (The results ot' the pulsed cold cathode source of Bolotin et $a 1^{44}$ shown in Table I cannot be compared with other sources since the method of measurement does not necessarily reflect the true fractions of ion currents produced in the source.)

c) Pulsing the discharge to obtain higher instantaneous arc powers is advantagenus. A direct comparison of pulsed and continuous operation at the same mean current can be made with the results quoted by Papineau et $a I^{45}$ shown in Table I. The mean output of the total ion beam was about the same in both cases. The selfheated cathode source 46 (Fig. 9) of Bennett performs well under continuous operation with the lighter ions but for argon and heavier elements is inferior to the pulsed sources. This is due to the inability to operate the arc at high voltages and currents even at the minimum gas flow. As explained previously, this could be the result of cathode material contributing to the gas pressure in the arc.

It is possible to produce multiply charged ions of any element. Gases are easily introduced into the source but solid materials are a little more difficult. It may be achieved by vaporizing the solid in a furnace or by sputtering the material with ions accelerated from the discharge. Alternately, a gaseous or volatile compound of the solid such as a fluoride may be used. Pasyuk et al 37, 47, 48, 49 have investigated a number of materials in P.I.G. sources and have obtained large currents of highly charged ions. With tungsten, ions up to $\mathrm{W}^{10+}$ have been obtained; using $\mathrm{WF}_{6}$ vapour $\mathrm{W}^{12+}$ ions were produced. Gavin 50 has also produced multiply charged fons of solid materials using sputtering. Bennett 46 has placed the solid in the discharge chamber where it is directly heated by the discharge. This method has been found satisfactory for a widc range of substances from lithium to uranium. Ions of $\mathrm{Ta} 10+$ and $\mathrm{U}^{11+}$ have been observed. 51

\section{Duoplasmatron Ion Sources}

The duoplasmatron source, developed by von Ardenne, 27 forms a hot cathode arc, with an intermediate electrode to both constrict the discharge and create an inhomogeneous magnetic field which concentrates the plasma near the extraction aperture in the anode. Very high ion current densities of up to several amperes per square centimeter of the aperture area per ampere of arc current are obtainable. The sources are frequently used in accelerators for the production of protons and singly charged ions.

However, it was not until quite recently that the source was developed for multiply charged ions, although as long ago as 1956, von Ardenne27 describes a source producing $\mathrm{N}^{3+}$ and $\mathrm{N}^{4+}$ ions. Brams et al, 52 using a standard commercial duoplasmatron source, found that at low gas pressures multiply charged ions of argon, krypton and xenon, but not helium and neon, were produced.

Extensive development of the duoplasmatron for multiply charged ions has been carried out by Illgen 53 at Heidelberg for a heavy ion linear accelerator. The source gcometry was found to be very important in obtaining the maximum yield of highly charged ions.

The data, shown in the table, indicates that the duoplasmatron is inferior to the P.I.G. source in the production of the higher charge states although the mean charge is quite high.

\section{Spark Sources}

Few sources of this type have been constructed for accelerators. Bolotin et al 54 describes a source for a linear accelerator. The discharge takes place in an aluminium nitride chamber, ions of this material being formed. About $30 \%$ of the $15 \mathrm{~mA}$ total current was nitrogen ions.

The short pulsed nature of the discharge is an obvious disadvantage. Plyutto et al 55 describes a source employing multiple spark gaps fired in succession to increase the effective pulse length. Large currents of $\mathrm{C}^{5+}, \mathrm{N}^{6+}$ and $\mathrm{O}^{6+}$ as well as $\mathrm{Cu}^{18+}$ and $\mathrm{sn}^{23+}$ ions are reported.

More recently Zwally 56,57 has analyzed the beam from a spark source ( $2 \mu \mathrm{s}$ duration). Ions such as $\mathrm{C}^{4+}$ and $\mathrm{Cu}^{24+}$ (or probably even higher chargc statcs) were obtained with an energy spread corresponding to the $10 \mathrm{kV}$ applied across the electrodes.

\section{"Burnout"}

The O.R.N.I. Modc II Hot-Electron Plasma known as Burnout, 58 (Fig. 10) bears some similarities to a P.I.G. discharge. An electron beam of $0.5 \mathrm{~A}$ from a hot cathode is fired into the cylindrical anodc and a discharge sct 
up as the electrons oscillate between the cathode and a reflector. The gas pressure is about $10^{-4}$ Torr. Two coils form a magnetic mirror field to contain the plasma. In this type of discharge a very hot electron plasma is formed with densities of about $10^{11} \mathrm{~cm}^{-3}$ and temperatures of tens of kilovolts.

The device was placed in the magnetic field of an $180^{\circ}$ magnetic spectrometer arrangement and the charge state distribution was analyzed. 59 The resolution was poor and the results (for argon and krypton) should be treated with caution. However, the device does show considerable promise.

\section{Laser Ion Sources}

Peacock and Pease 60 have suggested the use of a powerful pulsed laser to produce highly ionized particles. By using magnetic confinement of the iono tho very short. bursts could be turned into pulses up to about $1 \mathrm{~ms}$ long. A yield of $10^{13}$ ions/s with charge states between 10 and 20 is expected.

Faure et $a{ }^{6 I}$ have recently extracted and analyzed highly ionized beams of aluminium and iron, produced in a laser beam of $2 \times 10^{12} \mathrm{~W} / \mathrm{cm}^{2}$ intensity of $30 \mathrm{~ns}$ duration. Ion fluxes of $10^{14}$ ions $/ \mathrm{cm}^{2} \mathrm{~S}$ of $\mathrm{Al}^{+}$and $10^{12}$ ions $/ \mathrm{cm}^{2} \mathrm{~s}$ of $\mathrm{Alll}+$ were obtained. It is predicted that with a laser intensity of $10^{14} \mathrm{~W} / \mathrm{cm}^{2}, \mathrm{Al} 137, \mathrm{Fe} 24+$ and $v^{60+}$ could be obtained.

\section{Ion Containment Sources}

An intense magnetically focused electron beam is being used by Donets et al ${ }^{24}$ to produce highly charged ions in an ultrahigh vacuum (Fig. 11). The positive ions are trapped in the space charge of the beam and become highly charged as they are repeatedly ionized. Electrodes at a positive potential at the ends of the ionization chamber prevent ion loss along the electron beam. The device (EBIS) is hoped to be developed to give $2 \times 10^{13}$ ions $/ \mathrm{s}$ of $\mathrm{U}^{24+}$ for use in the large cyclotron ${ }^{3}$ being built in Dubna. The device is necessarily of a puised nature since after a certain interval of time the electron beam will become neutralized by the positive ions and will no longer act as a trap.

Up to now in a vacuum pressure of only $2 \times 10^{-8}$ Torr and with a product of electron beam flux and storage time of $2 \times 10^{17} \mathrm{~cm}^{-2}, \mathrm{C}^{5+}, \mathrm{N}^{6+}$ and $0^{7+}$ ions have been observed. When gold particles were injected into the apparatus, about $10^{8}-10^{9}$ ions/s of $\mathrm{Au}^{19+}$ were obtained.

Another device which showed great promise but on which work has ceased is the HIPAC source 20 (Fig. 12). An electron cloud is contained by a toroidal magnetic field and traps ions in the electrostatic potential well. The electrons of about $10 \mathrm{keV}$ energy rotate perpendicularly to the magnetic field. Vacuum pressures of about $10^{-10}$ Torr are required for containment times of several seconds necessary to produce $\mathrm{U}^{60+}$ with an electron flux of about $6 \times 10^{20}$ electrons $/ \mathrm{cm}^{2} \mathrm{~s}$. The yield of $\mathrm{u}^{60+}$ ions is estimated to be over $101 \mathrm{i}$ ions/s. Extraction of the ions is achieved by allowing them to leak out of a small negatively biased aperture in the chamber wall as the magnetic field is reduced. By slowly reducing the field, a fairly long beam pulse may be obtained (up to $50 \%$ duty cycle).

Highly charged ions are also being sought ${ }^{62}$ at the University of Maryland with a contained electron beam. The product of electron flux and containment time is $3 \times 10^{20}$ and could be increased to $3 \times 10^{21}$ electrons/

\section{Conclusions}

P.I.G. sources have been, and still are the main providers of highly charged heavy ions for accelerators. It is doubtful whether they can be significantly improved without some fairly major change. By extrapolation of existing data, one might expect at best to obtain small currents of $\mathrm{U}^{15+}$ ions.

With spark and laser sources it is quite clear that very highly charged ions are produced. The problems of the pulse length and extraction without loss of the higher charge states must be overcome before a source becomes a practical reality.

Probably the greatest promise is shown with the containment devices such as EBIS. In theory they appear to be capable of producing very high charge statcs and it is hoped that these projects will come to fruition in the not-too-distant future.

\section{References}

1. R.S. Livingston, Particle Accelerators 1, 51 (1970).

2. R.M. Main, IEEE Trans. NS-16, 791 (1969).

3. I.A. Shelaev, A. Zager, E.D. Vorobiev, S.I.Kozlov, V.I. Kuznetsov, R.Ts. Oganessian, Yu.Ts. Oganessian, K.I. Semin, A.N. Filipson, and V.A. Chugreev, Proceedings of this Conference, Paper E-19.

4. Ch. Schmelzer, IEEE Trans. NS-16, 788 (1969).

5. M.E. Rickey, M.B. Sampson and B.M. Bardin, IEEE Trans. NS-16, 396 (1969).

6. V.P. Sarantsev, to be published in the Proc. Int. Accel. Conf., Yerevan (1969).

7. H.A. Schwettman, Stanford University, private communication (1971).

8. V.S. Nikolaev and I.S. Dmitriev, Phys. Ietters 28A, 277 (1968).

9. H. Miessner, Nucl. Instr. and Meth. 72, 269 (1969).

10. K.D. Jenkins, Proc. Int. Cyclotron Conf., Oxford (1969).

11. F. Pleasonton and A.H. Snell, Proc. Roy. Soc. A241, 141 (1957).

12. B.I. Schram, F.J. de Hear, M.J. Van Der Wiel and J. Kistemaker, Physica 31, 94 (1965).

13. B.I. Schram, A.J.H. Boerboom and J. Kistemaker, Physica 32, 185 (1966) and B.L. Schram, Physica 32, 197 (1966).

14. Z.Z. Latypov, S.E, Kuprianov and N.N. Kunitskii, Sov. Phys.-JETP 19, 570 (1964).

15. P.A. Redhead, Can. J. Phys. 45, 1791 (1967).

16. L.J. Kieffer and G.H. Dunn, Rev. Mod. Phys. 38, I (1966).

17. W.B. Ard, R.A. Dandl, A.C. England, G.M. Haas and N.H. Lazar, Proc. Conf. on Plasma Physics and Coritrolled Nuclear Fusion Research, CuIham Iab., England (1965).

18. A. Zucker, Conf. on Heavy Ion Sources, USAEC Publication WASH-1159, Washington (March 1970).

19. H. Postma, Phys. Letters 31A, 196 (1970).

20. J.D. Daugherty, J.E. Eninger, G.S. Janes and R.H. Levy, lst Int. Conf. on Ion Sources, Saclay (June 1969).

21. T.H. Stix, Phys. Rev. Letters 23, 1093 (1969).

22. A.W. Trivelpiece, Conf. on Heavy Ion Sources, USAEC Publication WASH-1159, Washington (March 1970).

23. H.B. Williams, Phys. Rev. 107, 1451 (1957).

24. E.D. Donets, V.I. Ilyushchenko and V.A. Alpert, Ist Int. Conf. on Ion Sources, Saclay (June 1969).

25. See examples in Conference on Heavy Ion Sources, USAEC Publication WASH-1159, Washington (March 1970).

26. F.M. Penning, Physica 4, 71 (1937).

27. M. von Ardenne, Atomkern-Energie 4, 121 (1956).

28. B. Eden, Z. Astrophys. 22, 30 (1942).

29. T.N. Lie, Conf. on Heavy Ion Sources, USAEC Publication WASH-1.159, Washington (March 1970). 
30. D. Bohm, The Characteristics of Electrical Discharges in Magnetic Fields, eaited by A. Guthrie and R.K. Wakerling.

31. Yu.D. Pigarov and P.M. Morozov, Sov. Phys.-Tech. Phys. 6, 336 (1961) and 6, 342 (1961).

32. G.S. Navrogenes, W.J. Rambler, and C.B. Turner, IEEE Trans. NS-12, 769 (1965).

33. J.R.J. Bennett, lst Int. Conf. on Ion Sources, Saclay (June 1969).

34. J.R.J. Bennett, unpublished.

35. C.E. Anderson and K.W. Ehlers, Rev. Sc1. Instr. 27, 809 (1956).

36. R. MaIn, Conf. on Heavy Ion Suurces, USAEC Publication WASH-1159, Washington (March 1970).

37. A.S. Pasyuk, Yu.P. Tret' yakov and S.K. Gorbachev, Atomnaya Energiya 24, 21 (1968).

38. A.S. Pasyuk, Yu.P. Tret'yakov and V. Stanku, Prib. Tekh. Eksp. 3, 42 (1965).

39. R.J. Jones and A. Zucker, Rev. Sci. Instr. 25, 562 (1954).

40. P.M. Morozov, B.N. Makov and M.S. Ioffe, Atomnaya Energiya 3, 272 (1957).

41. F.I. Mineev and O. Kovpik, Prib. Tekh. Eksp. 8, $33(1963)$.

42. G. Nassibian, J.R.J. Bennett, D. Broadbent, S. Devons, R.W.R. Hoisington and V.E. Miller, Rev. Sci. Instr. 32, 1316 (1961).

43. B. Gavin, private communication (1971).

44. L.I. Bolotin, P.S. Markin and S.I. Meleshkov, Prib. Tekh. Eksp. 6, 86 (1961).

45. A. Papineau, P. Benezech and R. Maillard, J. Phys. Rad. 21, 410 (1960).

46. J.R.J. Bennett, to be published in the Proc. Int. Cyclotron Conf., Oxford (1969).

47. Yu.P. Tret' yakov, A.S. Pasyuk, L.P. Kul'kina and V.I. Kuznetsov, Atomnaya Energiya 28, 423 (1970), and J.I.N.R. - P7-4477, Dubna (1969).

48. A.S. Pasyuk, E.D. Vorob'ev, R.I. Ivannikov, V.I. Kuznetsov, V.B. Kutner, and Yu.P. Tret'yakov, Atomnaya Energiya 28, 75 (1970).

49. Yu.P. Tret' yakov, I.P. Kylkina, V.I. Kustnetsov and A.S. Pasyuk, J.I.N.R., P-7-5004, Dubna (1970).

50. B.F. Gavin, Nucl. Instr. and Meth. 64, 73 (1968).

51. J.R.J. Bennett, unpublished.

52. C.M. Braams, P. Zieske and M.J. Kofoid, Rev. Sci. Instr. 36, 1411 (1965).

53. J. Illgen, Unilac Group Report 4-68, Heidelberg.

54. L.I. Bolotin, P.S. Markin, Y.F. Kulygin, G.M. Skoromnii and S.I. Meleshkov, Prib. Tekh. Eksp. 6, 88 (1961).

55. A.A. Plyutto, K.N. Kervalidze and I.F. Kvartskhava, Atomnaya Energiya 3, 153 (1957).

56. H.J. Zwally, D.W. Koopman and T.D. Wilkerson, Rev. Sci. Instr. 40, 1492 (1969).

57. H.J. Zwally, Conf. on Heavy Ion Sources, USAEC Publication WASH-1159, Washington (March 1970).

58. I. Alexeff, R.V. Neidigh, W.F. Peed, E.D. Shipley and E.G. Harris, Phys. Rev. Letters 10, 273 (1963).

59. I. Alexeff, W.D. Jones and R.V. Neidigh, ORNI-TM2981 (April 1970).

60. N.J. Peacock and R.S. Pease, to be published in the Proc. Int. Cyclotron Conf., Oxford (Sept. 1969).

61. C. Faure, A. Perez, G. Tonon, B. Aveneau and D. Parisot, to be published in Phys. Ietters.

62. A.W. Trivelpiece, Conf. on Heavy Ion Sources, USAEC Publication WASH-1159, Washington (March 1970).

63. B.N. Makov, JINR Trans. of the Conf. on Nuclear Reactions with Multiply Charged. Ions, (1958) AECtr-4445.

64. R. Basile and J-M. Lagrange, J. Phys. Rad. 23, IIIA (1962).
65. K.W. Ehlers, Nucl. Instr. and Meth. 18, 19, 571 (1962)

66. K. Prelec and M. Isaila, to be published in Nucl. Instr, and Meth.

67. A. Ghiorso, R.M. Main and B.H. Smith, IEEE Trens. NS-13, 280 (1966).

68. A.S. Pasyuk and Y.B. Kutner, J.I.N.R.-P7-4289, Dubna (1969).

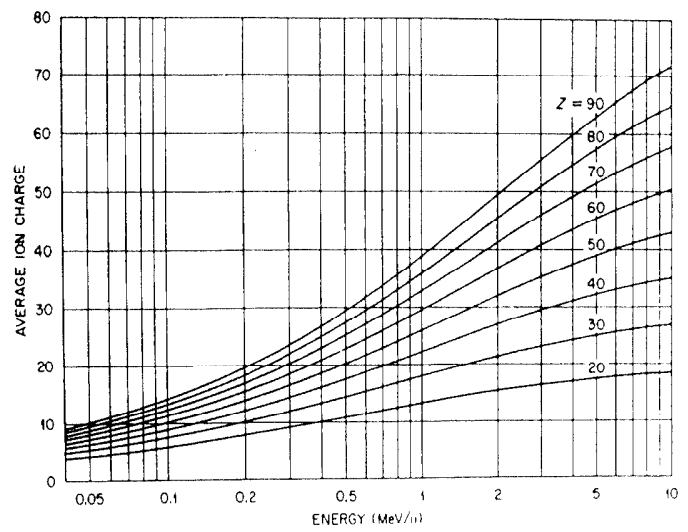

Figure 1 - Average equilibrium charge states, calculated from an empirical formula, ${ }^{8}$ or an ion beam passing through a solid stripper as a function of the energy/ nucleon and atomic number $z$ of the ion. Taken from Ref. 1 .

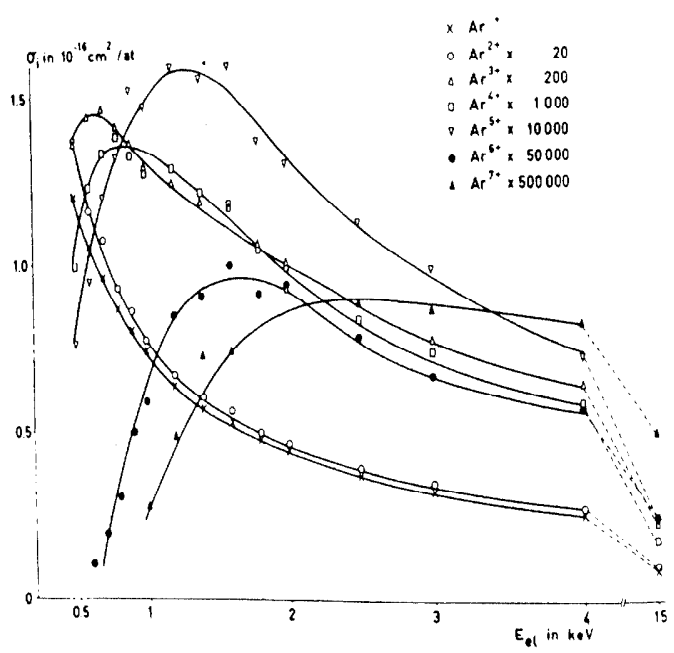

Figure 2 - Cross sections for production of mulliply charged argon ions in a single collision versus the incident electron energy. Taken from Ref. 13. 


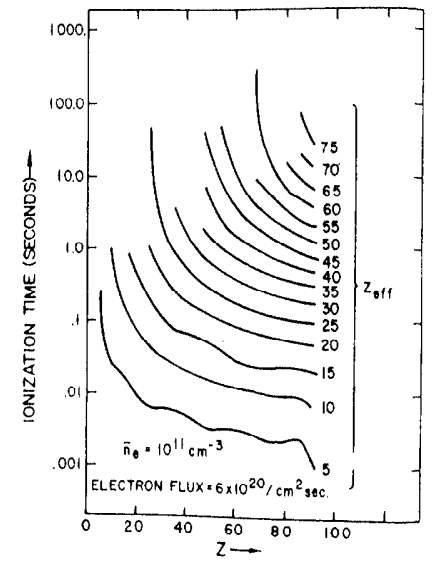

Figure 3 - Ionization times needed to create mean charge states $Z_{\text {eff }}$ for ions of atomic number $Z$. Taken from Ref. 20 .

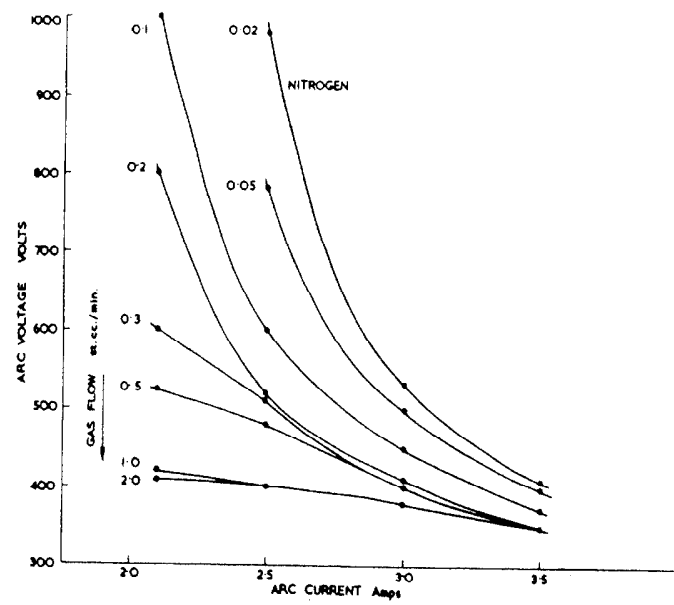

Figure it - Arc characteristics for a self (discharge) heated cathode. Taken from Ref. 33.

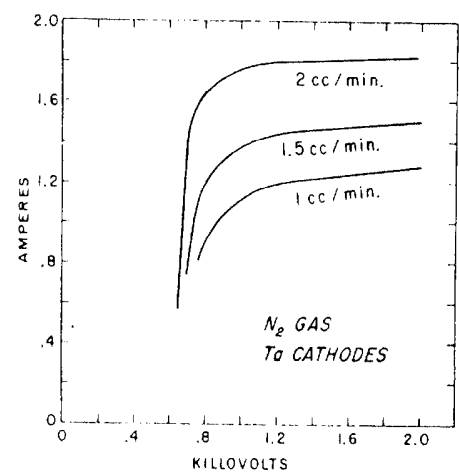

Figure 5 - Arc characteristics for a cold cathode P.I.G. source. Taken from Ref. 35.

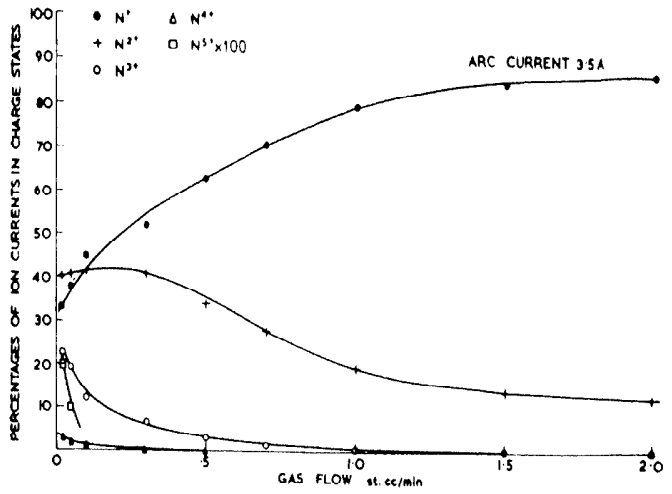

Figure 6 - Influence of gas flow on production of highly charged ions. Taken from Ref. 33.

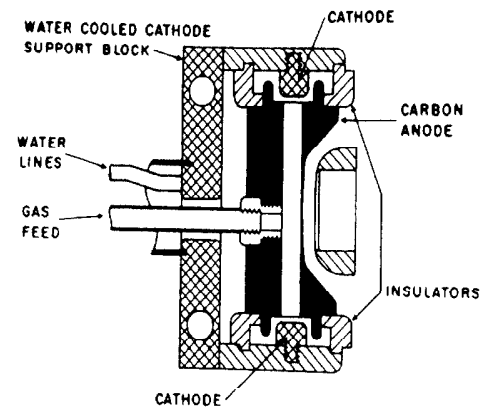

Figure 7 - Cold cathode P.I.G. Ion Source of Anderson and Ehlers. 35

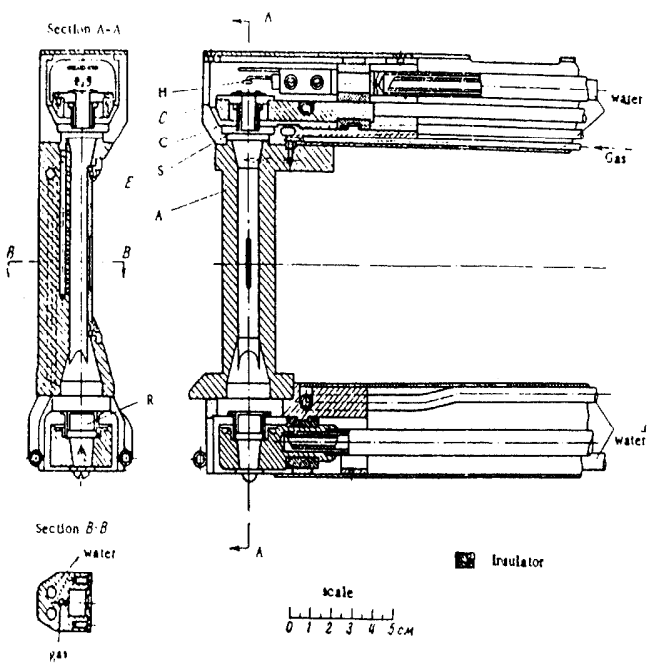

Figure 8 - Indirectly Heated Cathode P.I.G. Ion Source of Morozov, Makov and Ioffe. ${ }^{40}$ 


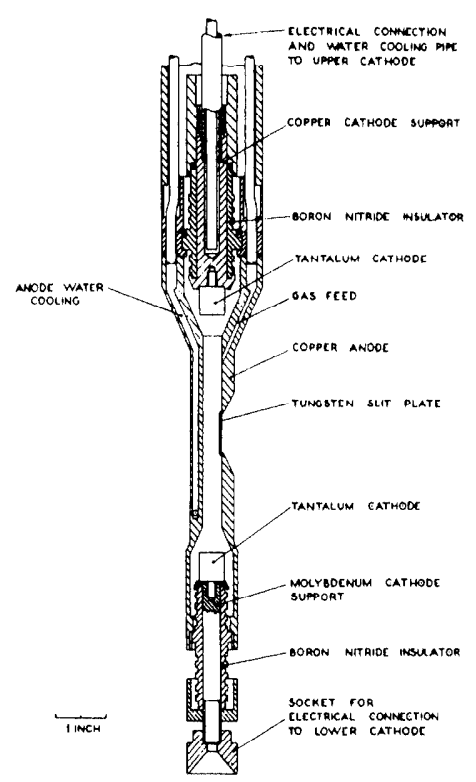

Figure $9-$ Self-Heated Cathode P.I.G. Ion Source of
Bennett 46

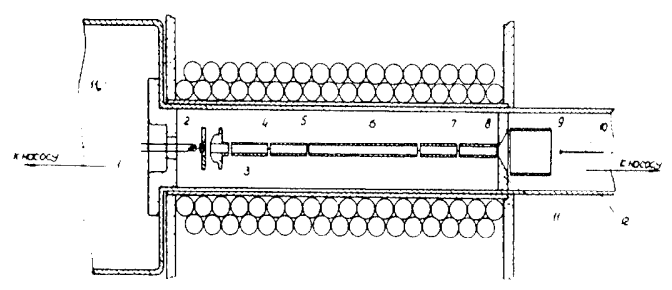

Figure 11 - The Electron Beam Source of Donets et a1. ${ }^{24}$

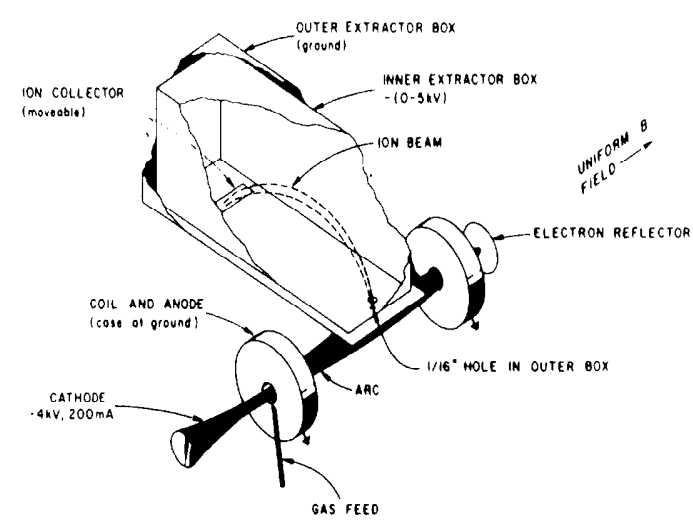

Figure $10-0 . R . N . L$. Mode It Hot Electron Plasma

("Burnout") and analyzer system. Taken from Ref. 59.

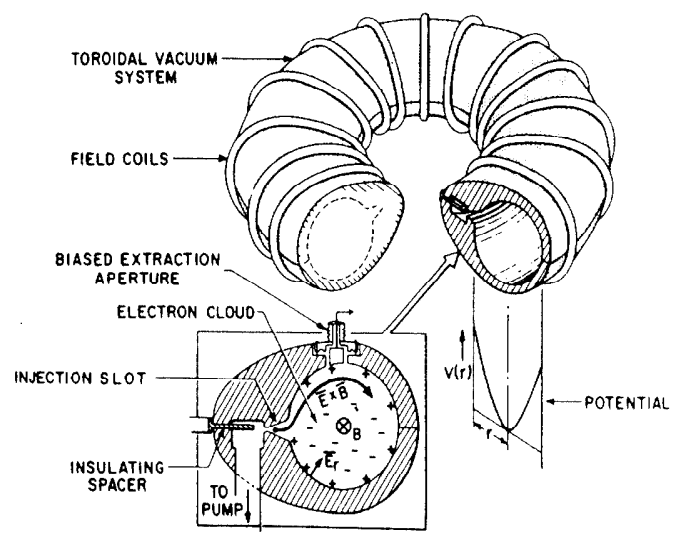

Figure 12 - The Hipac Ion Scurce. 20 


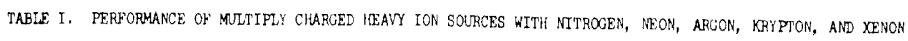

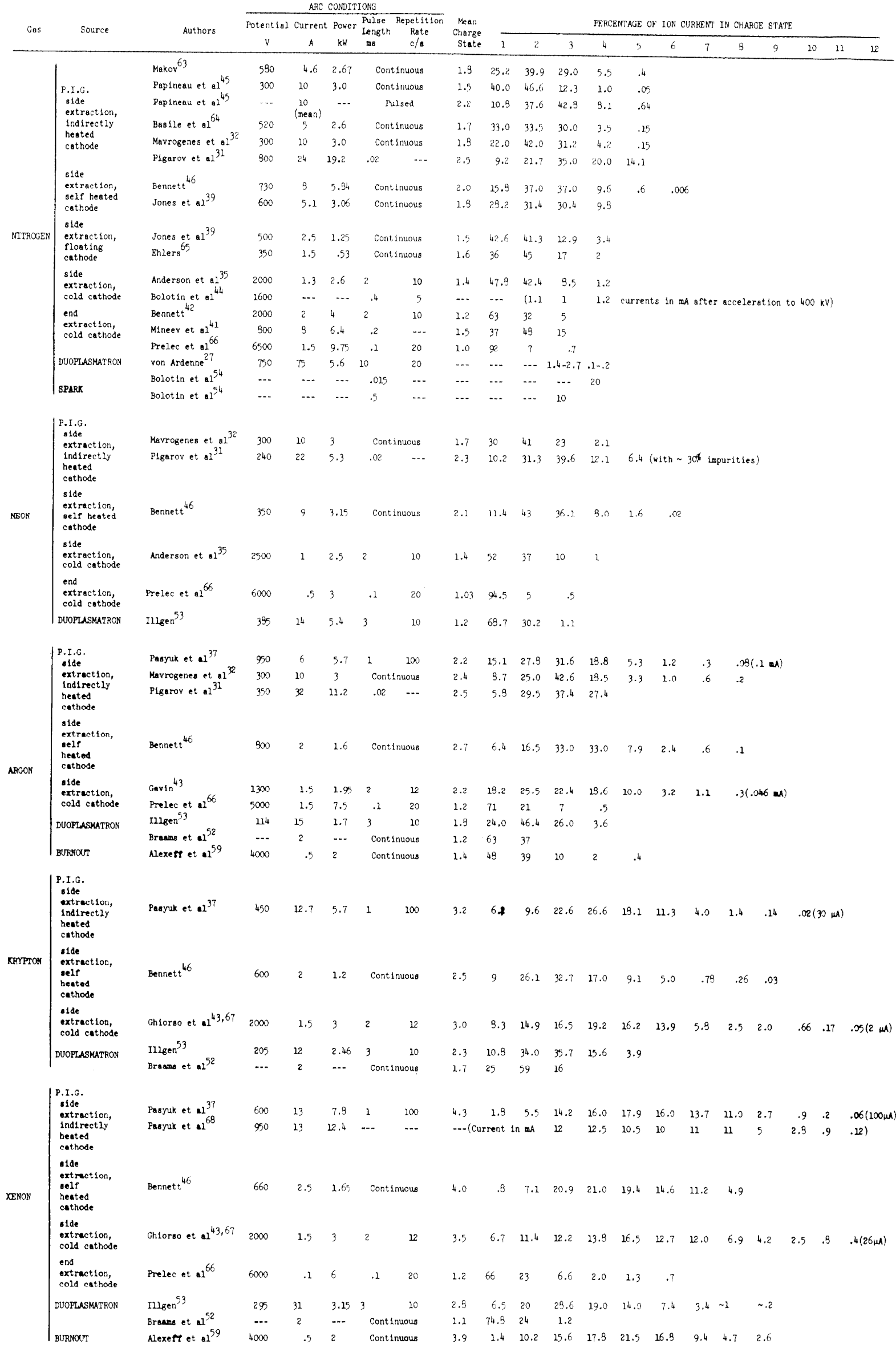

